\title{
Variables que inciden en la calidad educativa en un contexto de crisis sanitaria en instituciones educativas públicas de Comas
}

\section{Variables that affect educational quality in a context of health crisis in public educational institutions in Comas}

\author{
Isabel Menacho-Vargas \\ Universidad César Vallejo, Perú \\ ORCID: https://orcid.org/0000-0001-6246-4618 \\ Hugo Neptali Cavero-Ayvar \\ Universidad Andina Nestor Caceres Velasquez, Perú \\ ORCID: https://orcid.org/0000-0003-2161-4514 \\ Myriam Diana Orihuela Alvino \\ Universidad César Vallejo, Perú \\ ORCID: https://orcid.org/0000-0002-8745-5993 \\ Gisella Socorro Flores-Mejía \\ Universidad César Vallejo, Perú \\ ORCID: https://orcid.org/0000-0002-1558-7022
}

Received 10-12-20 Revised 11-25-20

Accepted 02-13-21 On line 02-27-21

*Correspondence

Email: isabelmenachov@gmail.com
Cite as:

Menacho, I; Cavero, H; Orihuela, M; Flores, G. (2021) Variables que inciden en la calidad educativa en un contexto de crisis sanitaria en instituciones educativas públicas de Comas. Propósitos y Representaciones, 9 (1), e1037. Doi: http://dx.doi.org/10.20511/pyr2021.v9n1.1037 


\section{Resumen}

Estudio cuantitativo, de tipo básica, diseño transeccionales correlaciones - causales, describe relaciones entre dos variables independientes, gestión del conocimiento y el liderazgo pedagógico y la variable dependiente calidad educativa. Tiene nivel explicativo y se ha realizado una síntesis de los aportes teóricos. Se aplicó los instrumentos a 112 docentes de una población de 181 docentes, la razón fue que se utilizó el Google formulario a través de un link que se envió a los docentes con la finalidad que pudieran acceder a las encuestas. Los instrumentos fueron 3 encuestas sobre calidad educativa, gestión del conocimiento y liderazgo pedagógico. Los mismos que cuentan con validez de contenido, para lo cual se utilizó la técnica de juicio de expertos, los mismo que han considerado que los instrumentos eran aplicables. Además, se obtuvo la confiabilidad de los instrumentos, a través del Alpha de Cronbach, en calidad educativa con 22 ítems, un coeficiente de ,928. Gestión del conocimiento con 22 ítems y un coeficiente de ,930 y en liderazgo pedagógico de 20 ítems con ,914, de coeficiente de confiabilidad. Los resultados encuentran un chi cuadrado de 17,311 y $\mathrm{p}$ valor de significancia de 0,002, la que está en los valores permitidos, inferiores al 0.05, por lo tanto, se demuestra la gestión del conocimiento y el liderazgo pedagógico incide en la calidad educativa en un contexto de crisis sanitaria en las instituciones educativas del distrito de Comas.

Palabras clave: igualdad y equidad en educación, gestión pedagógica, educación virtual.

\section{Abstract}

Quantitative study, basic type, cross-sectional correlations - causal design, describes relationships between two independent variables, knowledge management and pedagogical leadership, and the dependent variable educational quality. It has an explanatory level and a synthesis of the theoretical contributions has been made. The instruments were applied to 112 teachers from a population of 181 teachers, the reason was that the Google form was used through a link that was sent to the teachers in order to access the surveys. The instruments were 3 surveys on educational quality, knowledge management and pedagogical leadership. The same ones that have content validity, for which the expert judgment technique was used, the same ones who have considered that the instruments were applicable. In addition, the reliability of the instruments was obtained, through Cronbach's Alpha, in educational quality with 22 items, a coefficient of .928. Knowledge management with 22 items and a coefficient of 
.930 and in pedagogical leadership of 20 items with .914 , of reliability coefficient. The results find a chi square of 17.311 and $p$ value of significance of 0.002 , which is in the allowed values, lower than 0.05, therefore, it is demonstrated that knowledge management and pedagogical leadership affects educational quality in a context of health crisis in educational institutions in the district of Comas.

Keywords: equality and equity in education, pedagogical management, virtual education. 


\section{Introducción}

El año lectivo 2020 se convirtió para el mundo, América Latina y el Perú, en una situación de emergencia sanitaria por la Covid 19, lo que llevó a todos los sistemas educativos a la suspensión de clases presenciales; frente a esa situación las instituciones educativas tuvieron que utilizar diversas herramientas tecnológicas para crear ambientes de aprendizaje virtual de manera improvisada; y todo ello bajo la mirada atenta de la gestión directiva, Oliva (2020). La calidad educativa es el reflejo del esfuerzo que realizan los maestros en relación directa al apoyo de los directivos, los que realizan un trabajo de planeación, coordinación, administración, orientación, dirección y programación de la institución educativa, Bravo, Lora y Berastegui (2020). Ahora en un nuevo escenario como es el de la emergencia sanitaria y con una educación a distancia.

La educación es un bien público y a su vez un derecho fundamental para todos los ciudadanos, en este sentido la calidad y equidad son compromisos indiscutibles e ineludibles que los estados y las organizaciones internacionales asumen. Además, actualmente se afronta el reto de la calidad educativa, el mismo que ha sido ratificado en el Foro Mundial sobre la Educación efectuado en Incheon y considerado en la agenda 2030 para el desarrollo sostenible, UNESCO (2016).

La OCDE (2016), señala que en los países que tienen mejores resultados en la evaluación PISA, son los directivos los que aconsejan y orientan a los docentes para mejorar su práctica pedagógica centrados en el aprendizaje. En nuestro país, las evaluaciones nacionales e internacionales muestran la problemática, se revela un bajo desempeño del docente y del estudiante. En los resultados de PISA (2017), el Perú quedó en el puesto 64 de 70 países como lo señala la OCDE (2016) y en PISA (2017), demuestra un rendimiento por debajo al promedio, índice de desigualdad de los aprendizajes en las zonas urbano y rural, las bajas calificaciones de lectura, matemática y ciencias de los estudiantes.

Se realizan mediciones de forma permanente para conocer los niveles de calidad educativa empleando el SERVQUAL y en países donde los resultados son óptimos, las instituciones plantean realizar mejoras a dichas pruebas (Díaz y Troyano, 2016). Tal como sucede en países como China, Estados Unidos y España, donde se añaden nociones básicas centradas en las clases, además, las expectativas de los educandos en torno a su aprendizaje. En el Perú, las políticas educativas se centran en la gestión por resultados de aprendizaje, las instituciones educativas gestionan su propio cambio. SINEACE (2015), el Sistema Nacional de Evaluación, Acreditación y Certificación de la Calidad Educativa señala que la calidad educativa depende de diversos factores, entre 
los que destaca el rol del gestor de la institución educativa, que es el director y está relacionado con el liderazgo del docente e incide en el proceso de enseñar y aprender.

Los estudios realizados por Condori (2019), señalan la existencia de un nivel regular de liderazgo personal e institucional, lo que contribuye a alcanzar niveles regulares de gestión de conocimiento del capital intangible y de recursos humanos, alcanzando un resultado en la prueba estandarizada de Pearson. Núñez y Díaz (2017) en el estudio a directivos y docentes de instituciones educativos rurales y urbanas, utilizó la técnica la encuesta, el objetivo fue conocer las competencias gerenciales basado en los modelos de calidad en el contexto de la mejora continua, encontró que las competencias que deben desarrollar el personal directivo, es el manejo de dirección, el liderazgo y la gestión de la innovación.

En un estudio sobre la percepción de los docentes sobre el liderazgo, López, Fuentes \& Moreno (2018), encontraron que los líderes influyen en los docentes, motivando su praxis y la consecución de sus objetivos, a través de estrategias grupales como el trabajo en equipo, grupos de interaprendizaje, actividades sociales lo cual lleva a establecer vínculos afectivos. Para Maya, Aldana e Isea (2019), el liderazgo pedagógico busca beneficiar al proceso enseñanza aprendizaje, los investigadores aplicaron el coeficiente de correlación de Pearson, hallando 0.19 , encontrando relación entre el liderazgo y la calidad educativa

Niebles, Hernández \& Cardona (2016), investigó sobre la incorporación de herramientas tecnológicas para la gestión del conocimiento, en tanto, Avendaño, Paz, Parada-Trujillo (2016) estudiaron sobre la calidad educativa en instituciones públicas, sus hallazgos enfatizaron los aspectos administrativos, además, la calidad del aprendizaje, considerando el proceso de evaluación de los estudiantes, las prácticas innovadoras e investigación, el currículo de estudio y la autoevaluación de las instituciones.

Rivero, Hurtado y Morandé (2018) en su estudio con directores en Chile, hallaron que aproximadamente la tercera parte de los directores encuestados no contaba con experiencia en cargos de liderazgo escolar, además, que los directores evidenciaban debilidades en las funciones pedagógicas y de gestión. En cuanto Rodríguez, Ordoñez y López (2020), en España, realizaron un estudio con directores, encontrando que utilizan estrategías para desarrollo del liderazgo pedagógico a través de la labor colectiva, además, la muestra considera que participar en la acción pedagógica desarrollan su sensibilidad y compromiso con la labor. 
En un estudio sobre la comunicación y la difusión del proyecto educativo institucional, la comunicación efectiva, la visión y misión de una organización de educación se determinó que es fundamental para el liderazgo directivo, Castro, Flores, Cornejo \& Castro (2019), asimismo es común encontrar la falta de resolución de conflictos en las interacciones al interior de los espacios educativos. En esta línea de ideas, es fundamental el liderazgo pedagógico, relacionado al cumplimiento de metas y el compromiso del directivo con el proceso de enseñan, esto último lo enfatizan los investigadores, Santiago Espínola, Triveño, Guerrero y Martínez (2017).

Con respecto a la fundamentación teórica de calidad educativa, IPEBA (2013), es el mecanismo para llegar al máximo potencial y superar las expectativas propuestas. Murillo (2007), las instituciones que demuestran calidad se vinculan con cultura escolar, el trabajo dentro del aula cuyo objetivo es el aprendizaje de los estudiantes. Para MuñozRepiso y Murillo (2001), la calidad educativa se establece a partir de los fines educativos considerando los valores, la eficacia y la mejora continua del trabajo en las instituciones educativas.

La calidad educativa se conceptualiza en función a la eficacia, la relevancia y la interacción entre la concepción de educación, la cultura, la enseñanza y el rigor científico. Elola, N. y Toranzos, L (2000) y Vásquez (2013). En el mismo análisis, Avendaño, Paz \& Parada (2016), acuña componentes relacionados a la gestión pedagógica, como: planeación y diseño curricular y la gestión del talento humano. Y Martínera (2016), añade como factores relacionados a la calidad educativa, la gestión y administración del personal directivo, los que brindarán las condiciones de cobertura y equidad para los estudiantes.

La UNESCO (2016) propone los componentes de la calidad educativa: equidad, relevancia, pertinencia, eficacia y eficiencia. Lo que es desarrollado por otros autores como son, Poggi (2010) indica que la equidad, es impartir una educación de calidad para todos, teniendo en cuenta los aspectos culturales, geográficos e individuales de los educandos. Acerca de la relevancia, Pérez (2003), indica que se deben organizar las experiencias educativas en función al currículo de estudios de la EBR. Egido (2005), sobre la pertinencia es lograr los aprendizajes previstos en el currículo. Braslavsky (2006), la eficacia relacionado a la periodicidad y la eficiencia para Blanco (2008), está relacionado con la asignación y distribución de los recursos con los que se cuenta para el logro de los aprendizajes.

Con respecto a la gestión de conocimiento se encuentra relacionado con la dirección de los activos cognitivos favoreciendo la praxis pedagógica en los docentes, 
aporta en la indagación y permite obtener calidad en los procesos organizacionales. Araya, Salgado, Grandón, Ramírez \& Alfaro (2019). Para Rodríguez, Cohen, Pedraja, Araneda \& Rodríguez-Ponce (2014), es un conjunto de competencias esenciales, prácticas de saberes, habilidades de los miembros de las organizaciones. Tummapudi (2016), la gestión del conocimiento se vincula con la cultura de la innovación. Complementando, Martínez (2003) lo define como un proceso donde se gestiona los activos no materiales, teniendo como resultado mayor productividad y competitividad.

Otros teóricos como, Davenport \& Prusak (2001), indican que gestionar el conocimiento es desarrollar la competitividad. La gestión del conocimiento favorece la gestión interna de las instituciones educativas, permite la innovación, la mejora continua, la cultura organizacional y el desarrollo habilidades y destrezas del personal, además, mejora la toma de decisiones de los gestores, lo que trae como consecuencia, generar valor en la organización, Butnariu y Milosan (2012).

Para Nonaka y Takeuchi (1999), la gestión de conocimiento permite la producción de nuevos conocimientos. Además, se visibilizan y divulgan productos, servicios y sistemas. Por su parte, Davenport, De Long \& Beers (1997), señalan que gestionar el conocimiento es atraer, transferir y emplear los nuevos conocimientos.

Las dimensiones de gestión del conocimiento establecidas por Nonaka y Takeuchi (1995), están la creación de conocimientos, que promueve o reemplaza el cúmulo de conocimientos que ya se posee. Almacenamiento, se encuentra en bases de datos electrónicos y memorias. Transferencia, es la visibilidad del conocimiento, la dimensión aplicación y uso del conocimiento, es condensar el conocimiento en productos valiosos para la institución. También Tarí y García (2008), coincide con los mismos componentes y enfatiza que el buen manejo de los mismos, sirven para mejorar los resultados de la organización.

En cuanto al liderazgo pedagógico, York-Barr \& Duke (2004), afirman que los docentes conducen los aprendizajes de los estudiantes a partir de los procesos pedagógicos, fortaleciendo vínculos afectivos y de confianza. Para Bolívar (2010), es la capacidad de influir en los estudiantes motivándolos a la consecución de los objetivos educativos, agrega que el director tiene un rol transformador, instaura una cultura de cooperación y soporte psicológico.

Para Hallinger (2012), el liderazgo pedagógico se vincula al empleo de las estrategias metodológicas en el proceso de enseñar y aprender. Para Maureira (2004), el liderazgo pedagógico hace referencia a las habilidades de quien dirige el que se compromete con los objetivos, personas y cultura, en el caso de Robinson \& Lloyd \& 
Rowe (2014), enfatiza que el liderazgo pedagógico presenta una influencia al interior de las organizaciones de educación, la misma que permite alcanzar los propósitos pedagógicos.

El Minedu (2014), enfatiza que son los órganos directivos los que establecen las metas y expectativas, la medida de éxito es el resultado del aprendizaje, por lo tanto se debe enfatizar en el monitoreo. Maya, Aldana \& Argüelles (2019), existen índices de calidad cuando el personal directivo considere la planificación y la evaluación, además de una relación asertiva, vínculos afectivos, democracia y donde los integrantes de la institución se sientan en la confianza de participar. Y para Cuenca \& Cáceda (2017), los directores deben ser líderes pedagógicos con orientación organizacional y las funciones administrativas deben ser designadas a otros.

Para Robinson \& Lloyd \& Rowe (2014), las dimensiones son el establecer metas y expectativas, donde los lideres prioricen los propósitos educativos, considerando la metodología colaborativa. Otra dimensión, la obtención de recursos de manera estratégica, referido a la coherencia entre recursos y propósitos educativos. La retroalimentación posterior y observación del aula, considera la reflexión de la labor docente y su monitoreo permanente. El favorecer y participar en el aprendizaje y desarrollo de los docentes, el personal directivo se involucra con el quehacer pedagógico. La última, garantizar un ambiente ordenado y solidario, donde se priorice las buenas relaciones y el respeto.

En el estudio se presentan las siguientes interrogantes ¿De qué manera la gestión del conocimiento y el liderazgo pedagógico incide en la calidad educativa en los docentes de Comas?, los problemas específicos: ¿De qué manera la gestión del conocimiento, el liderazgo pedagógico incide en las dimensiones de la calidad educativa (equidad, relevancia, pertinencia, eficacia y eficiencia) de las organizaciones de educación de Comas?

El estudio se justifica porque presenta una revisión actualizada de la calidad educativa, gestión del conocimiento y liderazgos pedagógico proponiendo una independencia, contribuye a la teoría. A nivel metodológico, se presentan tres instrumentos y a nivel práctico se hacen llegar recomendaciones para lograr la calidad educativa a partir del trabajo de factores que inciden en ella.

El objetivo es determinar la incidencia de la gestión del conocimiento y el liderazgo pedagógico en la calidad educativa en un contexto de crisis sanitaria en de instituciones educativas públicas de Comas. De la misma forma, la hipótesis propone que la gestión 
del conocimiento y el liderazgo pedagógico incide en la calidad educativa en un contexto de crisis sanitaria en las instituciones educativas del distrito de Comas

\section{Método}

La investigación tiene el enfoque cuantitativo, de tipo básico, diseño transeccional correlacional - causal, describe las relaciones entre dos o más variables en un tiempo determinado, ya sea en términos correlaciones, o en función de la relación causa efecto, Hernández y Mendoza (2018). El nivel explicativo, según Sánchez y Reyes (2015), permite el descubrimiento de las variables causales que han podido incidir o afectar la ocurrencia de un fenómeno.

Se aplicó los instrumentos a 112 docentes de una población de 181 docentes, la muestra fue extraía por muestreo no probabilístico, intencionado, siendo siete instituciones educativas del distrito de Comas: I.E 2005 El Retablo, 23 docentes; I.E 2076 Abraham Lincoln, 18 docentes; IE 898-8193 Villas de Ancón, 18 docentes; IE 007 San Martin de Porres, 15 docentes; IE 2016 Francisco Bolognesi, 17 docentes; IE 2020 Maestro José Antonio Encinas, 11 docentes; IE 2022 Sinchi Roca, 12 docentes.

Se utilizó el Google formulario donde se ingresaron las preguntas y la misma permitió llenar una base de datos. Los cuestionarios fueron enviados a los docentes por medio de un link mediante el whatshapp. Fueron tres instrumentos, sobre calidad educativa, gestión del conocimiento y liderazgo pedagógico. Los mismos que cuentan con validez de contenido, para lo cual se utilizó la técnica de juicio de expertos, los mismo que han considerado que los instrumentos eran aplicables. Además, se obtuvo la confiabilidad de los instrumentos, a través del Alpha de Cronbach, en calidad educativa con 22 ítems, un coeficiente de ,928. Gestión del conocimiento con 22 ítems y un coeficiente de ,930 y en liderazgo pedagógico de 20 ítems con, 914 , de coeficiente de confiabilidad.

\section{Resultados}

Con respecto a los resultados encontramos que el modelo explica que las variables independientes, gestión del conocimiento y liderazgo pedagógico inciden en la variable dependiente calidad educativa, al obtenerse un chi cuadrado de 17,311 y p valor de significancia de 0,002, la que está dentro de los valores permitidos, inferiores al 0.05 , por lo tanto se rechaza la hipótesis nula (la gestión del conocimiento y el liderazgo pedagógico no incide en la calidad educativa en un contexto de crisis sanitaria en las instituciones educativas del distrito de Comas)

Tabla 1 
Ajuste de los modelos

\begin{tabular}{lllll}
\hline Modelo & $\begin{array}{l}\text { Logaritmo de la } \\
\text { verosimilitud }-2\end{array}$ & Chi-cuadrado & gl & Sig. \\
\hline $\begin{array}{l}52,434 \\
\text { Sólo intersección }\end{array}$ & 35,071 & 17,311 & 4 &, 002 \\
\hline Final
\end{tabular}

En relación a la bondad de ajuste se encuentra que el valor de chi-cuadrado de Pearson para el modelo es de 16,788 y el valor de la desvianza es de 13,811, con un $p$ valor de ,032 y 0,087 en cada caso, evidenciándose que tanto la gestión del conocimiento y el liderazgo inciden en la calidad educativa de manera moderada, en un contexto particular, por la crisis sanitaria debido al Covid 19.

Tabla 2

Bondad de ajuste

\begin{tabular}{llll}
\hline & Chi-cuadrado & gl & Sig. \\
\hline Pearson & 16,788 & 8 &, 032 \\
Desvianza & 13,811 & 8 &, 087 \\
\hline
\end{tabular}

Sobre la prueba del pseudo $\mathrm{R}$ cuadrado, los resultados predictivos determinados por el coeficiente de Nagalkerke, demuestran que la variabilidad de la calidad educativa se debe al $10.5 \%$ de incidencia de las variables gestión del conocimiento y liderazgo pedagógico, de lo que concluye que las variables independientes inciden en la variable dependiente calidad educativa, en un épocas de pandemia en profesores del nivel primaria del distrito de Comas.

Tabla 3

Pseudo R cuadrado.

\begin{tabular}{llll}
\hline & Cox y Snell & Nagelkerke & McFadden \\
\hline Resultado &, 080 &, 105 &, 065 \\
\hline
\end{tabular}

Los datos de la tabla de las estimaciones de parámetros se desanuda el valor $p$ de 0.000 en la intersección del inicio para la variable dependiente calidad educativa, de forma poco adecuado, obteniendose los valores de Wald de 33,086 siendo el p-valor de 0,000 menor.

Tabla 4

Estimaciones de parámetro

\begin{tabular}{lllll}
\multirow{2}{*}{ Estimación } & Desv. & Error $\quad$ Wald $\quad$ gl $\quad$ Sig. & Intervalo de \\
confianza al $95 \%$
\end{tabular}




\begin{tabular}{|c|c|c|c|c|c|c|c|c|}
\hline & & & & & & & $\begin{array}{l}\text { Límite } \\
\text { inferior }\end{array}$ & $\begin{array}{l}\text { Límite } \\
\text { superio } \\
r\end{array}$ \\
\hline \multirow[t]{2}{*}{ Umbral } & $\begin{array}{l}{[\mathrm{V} 3=} \\
1]\end{array}$ & $-4,073$ & ,534 & 58,272 & 1 & ,000 & $-5,119$ & $-3,027$ \\
\hline & $\begin{array}{l}{[\mathrm{V} 3=} \\
2]\end{array}$ & $-1,270$ & ,221 & 33,086 & 1 & ,000 & $-1,703$ &,- 837 \\
\hline \multirow{6}{*}{$\begin{array}{l}\text { Ubicació } \\
n\end{array}$} & {$[\mathrm{~V} 1=1]$} & 1,488 & 2,149 & ,480 & 1 & ,488 & $-2,723$ & 5,699 \\
\hline & {$[\mathrm{V} 1=2]$} &,- 073 & ,358 & ,042 & 1 & ,838 &,- 774 & ,628 \\
\hline & {$[\mathrm{V} 1=3]$} & $0^{a}$ & . & . & 0 & & . & . \\
\hline & {$[\mathrm{V} 2=1]$} & $-4,160$ & ,936 & 19,762 & 1 & ,000 & $-5,994$ & $-2,326$ \\
\hline & {$[\mathrm{V} 2=2]$} &,- 016 & ,385 & ,002 & 1 & ,966 &,- 770 & ,737 \\
\hline & {$[\mathrm{V} 2=3]$} & $0^{a}$ & . & & 0 & & & \\
\hline
\end{tabular}

a. Este parámetro está establecido en cero porque es redundante.

\section{Discusión}

Los resultados obtenidos con la regresión ordinal demuestran que los docentes perciben incidencia de la gestión del conocimiento y liderazgo pedagógico en la calidad educativa. Los hallazgos del comportamiento de la variable gestión del conocimiento se alinean a lo expresado por Nonaka y Takeuchi (1999), que indican que son los directivos los que gestionan la información para la creación de los conocimientos, favorecen la implementación de los proyectos de innovación, aunque tienen debilidades por la carencia de registros o informes para registrar las buenas prácticas, y recurrir a ellas cuando sea necesario. Además, Condori (2019) complementa resaltando la importancia del liderazgo del directivo en la imagen institucional, las relaciones interpersonales y el clima institucional.

Por el contexto de la pandemia y el confinamiento social decretado por el gobierno peruano desde marzo del 2020, para prevenir que la enfermedad por Coronavirus se propague, se evidencia mayor complejidad para realizar un trabajo participativo y de capacitación con los docentes, lo que afectó en la socialización de los proyectos de innovación, además la toma de decisiones, situación que coincide por lo vertido por Avendaño, Paz y Parada-Trujillo (2016), quienes afirman que la escuela es un escenario educativo donde se realiza trabajo participativo.

El estudio de Araya, Salgado, Ramírez y Alfaro (2019), demuestra que la gestión del desarrollo organizativo en las escuelas afecta significativamente en los diversos procesos que cumplen, lo que conlleva a conocer la disponibilidad y utilización adecuada 
del conocimiento que existe para el desenvolvimiento de las acciones de estas organizaciones.

Poggi (2010), afirma que la educación es un derecho fundamental, prioriza el respeto a las particularidades del educando en el aspecto cultural, étnico y social, asumiendo una postura de calidad educativa, en esta línea de ideas los hallazgos del estudio aportan demostrando que el valor del modelo tiene una significancia de $0.000 \mathrm{y}$ el pseudo cuadrado predictivo determinado por el coeficiente de Nagalkerke, demuestran que la variabilidad de la calidad educativa se debe al $10.5 \%$ de incidencia de las variables gestión del conocimiento y liderazgo pedagógico en época de pandemia en docentes del nivel primaria del distrito de Comas.

Las condiciones actuales de educación virtual o remota, exigen a las instituciones educativas y a los gestores generar las condiciones de calidad que los estudiantes necesitan, para lo cual, el órgano estatal responsable es el IPEBA (2013), quien hace referencia a los mecanismos para llegar al máximo potencial y superar las expectativas propuestas, las que están relacionadas con el liderazgo y como se lleva la gestión de conocimientos en las instituciones. También la calidad educativa se relaciona con la cultura escolar, según Murillo (2007). La calidad educativa se establece a partir de los fines educativos y la mejora continua de cada miembro de la comunidad educativa. Muñoz-Repiso y Murillo (2001).

El estudio demuestra que el liderazgo incide en la calidad, por lo concuerda con Martínera (2016) quien afirma que se logra la calidad educativa con la gestión y liderazgo que ejercen los directivos, que son quienes plantean la prospectiva y vida institucional. Araya, Salgado, Grandón, Ramírez \& Alfaro (2019), hacen referencias con la dirección de los activos cognitivos y favorecen los procesos organizacionales, en esto coinciden con Rodríguez, Cohen, Pedraja, Araneda \& Rodríguez-Ponce (2014), mientras, Tummapudi (2016), se suma la gestión del conocimiento vincula a la calidad con una cultura de la innovación, lo que permite resultados de mayor competitividad y productividad, Martínez (2003).

Los resultados del estudio, también indican que la gestión del conocimiento incide en la calidad educativa, lo que fundamenta por su relación con mayores niveles de competitividad, gestión interna, innovación, aporta en la cultura organizacional y en la mejora continua, en esto coiciden, Davenport \& Prusak (2001) y Butnariu y Milosan (2012). 


\section{Conclusiones}

Los resultados predictivos determinados por el coeficiente de Nagalkerke, demuestran que la variabilidad de la calidad educativa se debe al $10.5 \%$ de incidencia de las variables gestión del conocimiento y liderazgo pedagógico, lo que permite concluir que las variables independientes inciden en la variable dependiente calidad educativa, en un épocas de pandemia en profesores del nivel primaria del distrito de Comas.

Los instrumentos aplicados han demostrado una pobre percepción de los profesores del nivel primaria del distrito de Comas, con respecto a la Gestión del conocimiento, Liderazgo pedagógico y Calidad educativa en un contexto de aislamiento social por la Covid 19, situación que se agudizo al pasar a una educación virtual.

En posteriores estudios se debe considerar estrategias y buenas practicas en torno a las variables independientes de este estudio, gestión del conocimiento y liderazgo pedagógico, cuya implementación permita alcanzar la calidad educativa que constituye un derecho fundamental para los educandos.

\section{Referencias}

Araya, S. Salgado C., Grandón, E. Ramírez, P.\& Alfaro, J. (2019) Explorando la relación entre gestión del conocimiento y rendimiento organizativo en organizaciones educativas. Iberian Conference on Information Systems and Technologies (CISTI), Coimbra, Portugal, 2019, pp. 1-5.

Avendaño, W., \& Paz, L., \& Parada-Trujillo, A. (2016). Estudio de los factores de calidad educativa en diferentes instituciones educativas de Cúcuta. Investigación \& Desarrollo, 24(2),329-354. [fecha de Consulta 2 de Julio de 2020]. ISSN: 0121-3261. Disponible en:

Bolívar, A. (2010). El liderazgo educativo y su papel en la mejora: Una revisión actual de sus posibilidades y limitaciones. Psicoperspectivas. Individuo y Sociedad, 9(2), 9-33. doi:10.5027/psicoperspectivas-vol9-issue2-fulltext-112.

Blanco, R. (2008). Eficacia escolar desde el enfoque de calidad de la educación. En R. Blanco, I. Aguerrondo, G. Calvo, G. Cares, L. Cariola, R. Cervini, N. Dari, E. 
Fabara, L. Miranda, F. J. Murillo, R. Rivero, M. Román y M. Zorrilla (Eds),

Eficacia escolar y factores asociados en América Latina y el Caribe (pp. 7-16).

Santiago de Chile, Chile: UNESCO/LLECE.

Braslavsky, C (2006). Diez factores para una educación de calidad para todos en el siglo XXI. Fundación Santillana. Madrid.

Braslavsky, C (2012). Diez factores para una educación de calidad para todos en el siglo XXI, REICE. Revista electrónica sobre calidad eficacia y cambio en educación.

Butnariu, M y Milosan, L (2012). Evaluación preliminar de la gestión del conocimiento en las universidades. Ciencias sociales y del comportamiento, Elsevier. 62 (2012) 791 - 795. doi: 10.1016 / j.sbspro.2012.09.133

Bravo, E., Lora, L. y Berastegui, Y. (2020). Neuroliderazgo como estrategia para el fortalecimiento de la gestión directiva en instituciones educativas. Actualidades Investigativas en Educación, 20 (1), 29-29.

Castro, S., Flores, F., Cornejo, C., Castro, M. (2019) Competencias de Liderazgo que movilizan docentes en puestos directivos escolares de establecimientos educativos particulares subvencionados de la $\mathrm{V}$ Región de Chile. Revista Educación. 43(2). http://dx.doi.org/10.15517/revedu.v43i2.31834

Condori, Y. (2019). Liderazgo y gestión del conocimiento de los directivos del Instituto Superior Pedagógico Público de Puno. Revista Innova Educación, 1(2), 189196.DOI: https://doi.org/10.35622/i.rie.2019.02.005

Cuenca, R., Cáceda, J. (2017). Ideales Normativos, Normas y Praxis: Patologías Sociales sobre los Directivos Escolares en el Perú. REICE. Revista Electrónica Iberoamericana sobre Calidad, Eficacia y Cambio en Educación, 2017, 15(2).

Davenport, T., De Long, D.., \& Beers, M. (1997). Building successful knowledge management projects. Center for business innovation working paper, 4 . 
Davenport, T. H., \& Prusak, L. (2001). Conocimiento en acción como las organizaciones manejan lo que saben. Prentice Hall.

Díaz, J., y Troyano, Y. (2016). La gamiicación en las Aulas. Revista INED 21. Recuperado de: https://ined21.com/la-gamiicacion-las-aulas/

Egido, I. (2005). Reflexiones en torno a la evaluación de la calidad educativa. Tendencias pedagógicas, 10, 17-28.

Elola, N. y Toranzos, L (2000). Evaluación educativa: una aproximación conceptual. Barcelona: OEI en http://www.oei.es/calidad2/luis2.pdf Noviembre 2011.

Espínola, V., Treviño, J., Guerrero, M., \& Martínez, J. (2017). Liderazgo para la mejora en escuelas vulnerables: prácticas asociadas al cumplimiento de metas de aprendizaje. Estudios pedagógicos (Valdivia), 43(1), 87106. https://dx.doi.org/10.4067/S0718-07052017000100006

Hallinger, P. (2012). School Leadership that Makes a Difference: Lessons from 30 Years of International Research [Liderazgo de la escuela que hace: Lecciones de diferencia a partir de 30 años de investigación internacional]. Roma: Ministry of Education

Hernández, R. y Mendoza, C. (2018). Metodología de la Investigación: Las rutascuantitativa, cualitativa y mixta. México: Mc Graw Hill.

IPEBA. (2013). Qué y cómo evaluamos la gestión de la institución educativa (2da ed.). Lima, Perú: Ministerio de Educación

López, J., Fuentes, A. y Moreno, A. (2018). El liderazgo efectivo en los centros concertados de naturaleza cooperativa: Percepciones de sus docentes. Actualidades Investigativas en Educación, 18(3), 318-339. doi: https://revistas.ucr.ac.cr/index.php/aie/article/view/34195.

Maureira, O. (2004). Liderazgo factor de eficacia escolar, hacia un modelo causal. Revista Electrónica Iberoamericana sobre Calidad, Eficacia y Cambio en Educación. 2(1), 1-20. Recuperado de http://www.ice.deusto.es/rinace/reice/vol2n1/Maureira.pdf. 
Ministerio de Educación Perú. (2014). Marco de buen desempeño del directivo. Lima: Ministerio de Educación del Perú.

Martínera, V. (2016). Orientaciones de políticas educativas del Consejo de Educación Inicial y Primaria. España: Consejo de Educación Inicial y Primaria. Obtenido de http://www.ceip.edu.uy/documentos/2017/ceip/OpeCEIP2016-2020.pdf

Martínez, E. (02 de 2003). Gestión del conocimiento. Obtenido de http://www.bizkaia.eus/:http://www.bizkaia.eus/Home2/Archivos/DPTO8/Tem as/Pdf/ca GTcapitulo2.pdf?hash=51ad832f2ef685c02d6feb7768c61887

Maya, E., Aldana J., \& Argüelles, I. (2019). Liderazgo Directivo y Educación de Calidad. CIENCIAMATRIA, 5(9),

Maya, E., Aldana, J. \& Isea, J. (2019). Liderazgo Directivo y Educación de Calidad. CIENCIAMATRIA.

Muñoz-Repiso, M. y Murillo, F.J. (2001). Un balance provisional sobre la calidad en educación: eficacia escolar y mejora de la escuela. Organización y Gestión Educativa, 9(4), pp. 3-9. https://www.redalyc.org/pdf/551/55114080011.pdf

Murillo, F. J. (2007). Investigación iberoamericana sobre eficacia escolar. Bogotá: Convenio Andrés Bello.

Niebles, W., Hernández, H., \& Cardona, D. (2016). Gestión tecnológica del conocimiento: herramienta moderna para la gerencia de instituciones educativas. REVISTA DE INVESTIGACIÓN, DESARROLLO E INNOVACIÓN, 7(1),

36. https://doi.org/10.19053/20278306.v7.n1.2016.5633

Nonaka, I., Takeuchi, H. (1995). La organización creadora de conocimiento. Cómo las compañías japonesas crean la dinámica de la innovación (M. H. Kocka, Trans. 1 ed.). México: Oxford University Press. 
Nonaka \& Takeuchi (1999). La organización creadora de conocimiento. México, D.F.:Oxford University Press México. Recuperado de http://www.bnp.gob.pe/documents/transparencia/mof/org linea/dtbnp/c $\underline{\text { bn/cbn.pdf }}$

Núñez, N., Díaz, D. (2017). Perfil por competencias gerenciales en directivos de instituciones educativas. Estudios pedagógicos (Valdivia), 43(2), 237252. https://dx.doi.org/10.4067/S0718-07052017000200013

OCDE (2016). Panorama de la educación. Indicadores OCDE 2016. http://educalab.es/inee/indicadores-educativo/informe-2016

Oliva, H. (2020). La educación en tiempos de pandemia: visión desde la gestión de la educación superior. Disruptiva. Periodismo, Ciencia y Tecnología. Número ISSN. 2706-542. Universidad Francisco Gavidia.

Organización para la Cooperación y el Desarrollo Económicos, OCDE. (2000). Knowledge Management in the learning society. Recuperado de http://ocw.metu.edu.tr/file.php/118/Week11/oecd1.pdf

Parada-Trujillo, A. (2016). Estudio de los factores de calidad educativa en diferentes instituciones educativas de Cúcuta Investigación \& Desarrollo, vol. 24, núm. 2, 2016, pp. 329-354 Universidad del Norte Barranquilla, Colombia.

Pérez, O. (2003). Alternativas para mejorar la calidad de la educación básica en el estado Táchira. [En línea] Disponible en: http://hdl.handle.net/10803/8898.

PISA (2017). Objetos interactivos de aprendizaje para la formación y evaluación competencial.

https://proyectodescartes.org/competencias/materiales PISA 2017.htm

Poggi, R. (2010). La gestión de un nuevo sistema educativo en función de la escuela. Educar. Revista de Educación. Recuperado de: http://www.oei.es/metas2021/CALIDAD.pdf

Rivero, R., Hurtado, C., \& Morandé, Á. (2018). ¿Cuán preparados llegan los directores escolares?: un análisis sobre su formación y trayectorias laborales previas a ejercer su cargo. Calidad en la Educación, (48), 17-49. 
Robinson, V., \& Lloyd, C., \& Rowe, K. (2014). El impacto del liderazgo en los resultados de los estudiantes: Un análisis de los efectos diferenciales de los tipos de liderazgo. REICE. Revista Iberoamericana sobre Calidad, Eficacia y Cambio en Educación, 12 (4), 13-40. Recuperado de http://www.redalyc.org/pdf/551/55131688002.pdf

Rodríguez, M., Ordóñez, R. y López, A. (2020). La dirección escolar: Liderazgo pedagógico y mejora escolar. Revista de Investigación Educativa, 38(1), 275292. https://doi.org/10.6018/rie.364581

Rodríguez E., Cohen, W., y otros tres autores. La gestión del conocimiento y la calidad de la docencia de postgrado en las universidades: un estudio exploratorio, doi: 10.15446/innovar.v24n52.42506, Innovar, 24 (52), 59-66 (2014)

Sánchez, H. y Reyes, C. (2015). Metodología y Diseños en la Investigación Científica. Lima: Business Support Aneth S.R.L.

SINEACE. Sistema Nacional de Evaluación, A. y. C. d. I. C. E. (2015). Congreso internacional "Calidad del ejercicio profesional en el Perú". Aportes de la certificación de competencias y nuevos desafíos. Compendio de exposiciones.

Tarí J. \& García M. (2008). Dimensiones de la gestión del conocimiento y de la gestión de la calidad: Una revisión de la literatura Investigaciones Europeas de Dirección y Economía de la Empresa. Volume 15, Issue 3, 2009, Pages 135148

Tummapudi N. (2016) Linking knowledge management and innovation culture for business performance improvement https://www.researchgate.net/publication/309856516

Unesco (2016). Panorama regional: América Latina y el Caribe. Recuperado de http://en.unesco.org/gem-report/sites/gem-report/files/191433s.pdf

Vasquez, A. (2013). Calidad y calidad educativa: Investigación educativa, 17 (02), 4971. 
https://revistasinvestigacion.unmsm.edu.pe/index.php/educa/article/view/820 $\underline{6 / 7157}$

York-Barr, J. y Duke, K. (2004). «What do we know about teacher leadership? Findings from two decades of scholarship». Review of Educational Research, 74(3), pp. 255-316 